\title{
SWEPT CONICS: Single Mirror Transformers
}

\author{
Paul K. Manhart ${ }^{\mathrm{a}}$, K. Scott Ellis ${ }^{\mathrm{b}}$ \\ ${ }^{a}$ NASA Langley Research Center, Remote Sensing Flight Systems Branch (D204), Mail Stop 468, Bldg \\ 1202A, Room 241A, 5 North Dryden Street, Hampton, VA 23681-2199 \\ ${ }^{\mathrm{b}}$ Photon Engineering LLC, 440 S. Williams Blvd., Suite 106, Tucson, AZ 85711
}

\begin{abstract}
A class of optical surfaces called 'Swept Conics' or 'Conical Spheroids' can be used to transform wavefronts from point sources to diffraction limited line or arc images, and vice versa.
\end{abstract}

Key Words: Optical design, swept conics, rotational symmetry, conical spheroids, single mirror transformers, conics of rotation

\section{Introduction:}

Traditional line imaging systems with zero etendue sources are based upon the use of homogeneous refractive lenses with cylindrical surfaces. Basic optical principles lead to the use of multiple elements in a lens system to provide imagery across a required field with acceptably low aberration content. The accurate fabrication and testing of polished cylindrical surfaces is difficult and costly compared to spherical surfaces. Making an achromatic cylindrical optical assembly can again add significantly to the cost and complexity.

There is a class of reflective surfaces that are ideally suited for transforming a point source into a line image, and vice-versa. The author refers to these as 'swept conics', because they are created by sweeping a conic section about a fixed axis that intersects one of the conics foci. For example, in Figures 1a - 1d, a Point-to-Line Generator can be created by sweeping an off-axis parabolic segment about an axis that passes through its focal point and is perpendicular to the optical axis of the parent parabola. In this example, a point source located at the parabolic focus is transformed into a line image located on the sweep axis, just above the point source. From the side, it looks like a point source is being collimated by an off-axis parabolic segment. From the top, it looks like a point source, located at the center of curvature of a circle, is being imaged on to itself. Since both views are diffraction limited, the transformation is free of all aberrations. Being reflective, the line image is achromatic as well. More examples can be found using other conical shapes such as elliptical and hyperbolic segments.

\subsection{Discussion}

This paper will show how to design these fascinating reflectors and show some of the predicted performance characteristics. Finally, we shall give some examples of how they might be used in optical and non-optical systems. One point of interest in all of the designs presented here is how they work in reverse. The Point-to-Line Generator for example, will form a diffraction limited line image when the object is a point source. Will it work in reverse? Will it form an Airy disc from a diffraction limited line source? The answer is 'Yes', with restrictions. One must pay close attention to the $\mathrm{X}$ and $\mathrm{Y}$ numerical aperture (NA) of the imaged line or arc from a point source with a symmetrical numerical aperture. The images in these devices are typically formed with a numerical aperture only in one dimension. So a line source will only image to a point if its YZ NA is zero and its XZ NA is finite

\footnotetext{
a paul.k.manhart@nasa.gov: phone +1.757.864.6441

b scotte@photonengr.com : phone +1.520.733.9557
} 


\subsection{Point-to-Line Generator}

As described in the Introduction, transforming a point source into a diffraction limited line requires an off-axis parabolic segment rotated about an axis that passes through the parabolic focal point and is perpendicular to the optical axis of the parent parabola. Figures 1a through 1d show the basic layout of such a device. Fabrication of such a mirror can be achieved on a diamond turning lathe where the axis of symmetry ( $\mathrm{Y}$ axis) is co-linear with the spindle axis of the lathe. The tool then runs in and out along the spin axis to generate the off-axis parabolic segment desired.

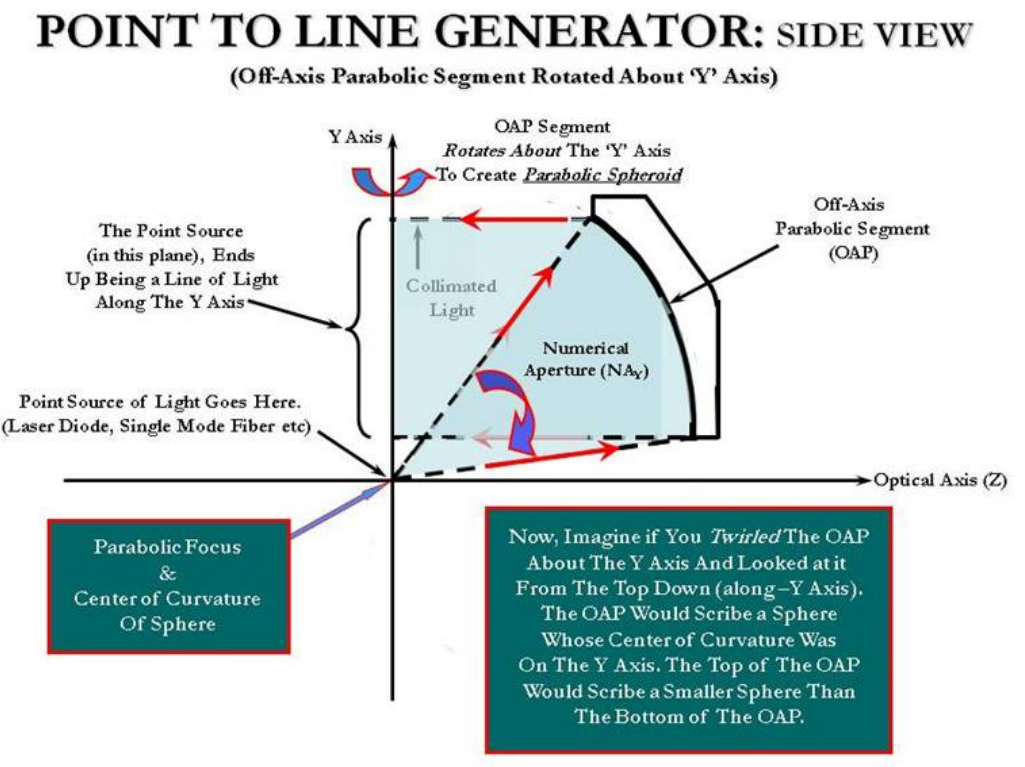

Figure 1a: SIDE View: Point-to-Line Generator: From the side, it looks like a point source is located at the focal point of an off-axis parabola. Light is collimated as it reflects off the mirror.

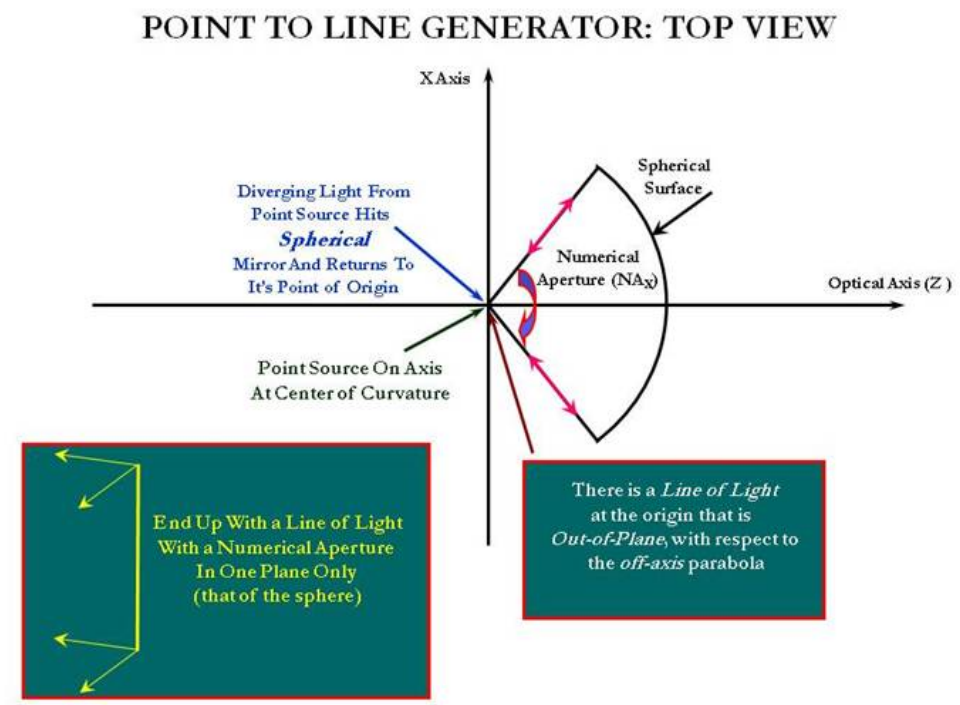

Figure 1b. TOP View: Point to Line Generator. From the top, it looks like a point source located at the center of curvature of a circle. Light from the point source is imaged right back on to itself in this flattened view. Note that in both side and top views, diffraction limited imagery is achieved. 

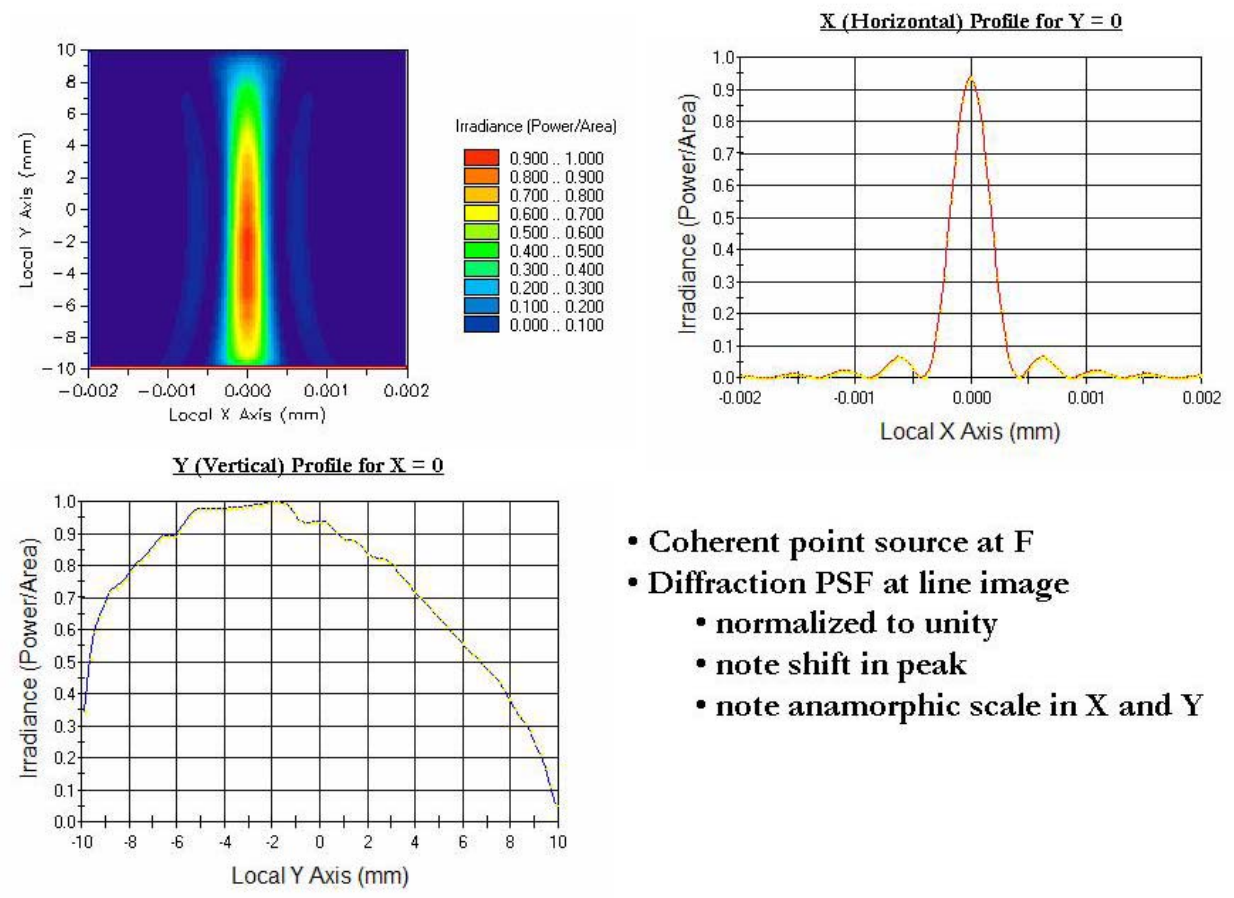

- Coherent point source at $\mathrm{F}$

- Diffraction PSF at line image

- normalized to unity

- note shift in peak

- note anamorphic scale in $\mathrm{X}$ and $\mathrm{Y}$

Figure 1c. With a coherent point source located at F, the PSF at the line image shows a diffraction limited width (along the $\mathrm{X}$ axis), and a linear dimension along the $\mathrm{Y}$ axis. Note the non-uniform illumination along the length of the line due to the non-uniform distance from source to mirror in the vertical direction.
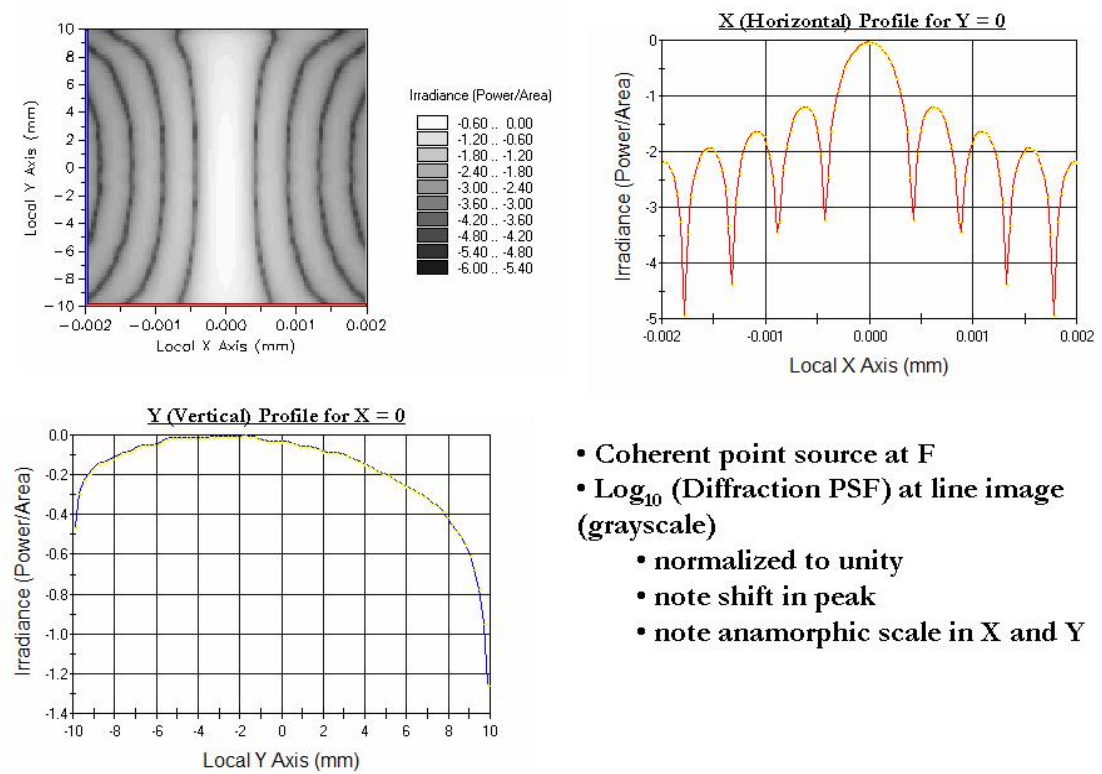

- Coherent point source at $\mathrm{F}$

- $\log _{10}$ (Diffraction PSF) at line image (grayscale)

- normalized to unity

- note shift in peak

- note anamorphic scale in $\mathrm{X}$ and $\mathrm{Y}$

Figure 1d. Log plot of PSF across the width of the line image 


\subsection{Point-to-Arc Generator}

In Figures 2a, 2b and 2c we create a Point-to-Arc relay by sweeping an elliptical segment about an axis that passes through one of the ellipse foci. Figure $2 \mathrm{a}$ and $2 \mathrm{~b}$ shows both the side and top view of such a surface. Notice that the axis of rotation passes through the elliptical segment whereas in the Point-to-Line Generator, the axis did not pass through the optical surface. This creates some interesting differences between the two, AND in the actual solid model itself. One consequence of having the rotation axis pass through the optical surface is that it is no longer possible to rotate the segment through a complete $2 \pi$ circle. At the intersection point of the rotation axis and the surface, the 3D model reduces down to a single point as all the infinitely thin segments pass through the same point in space.

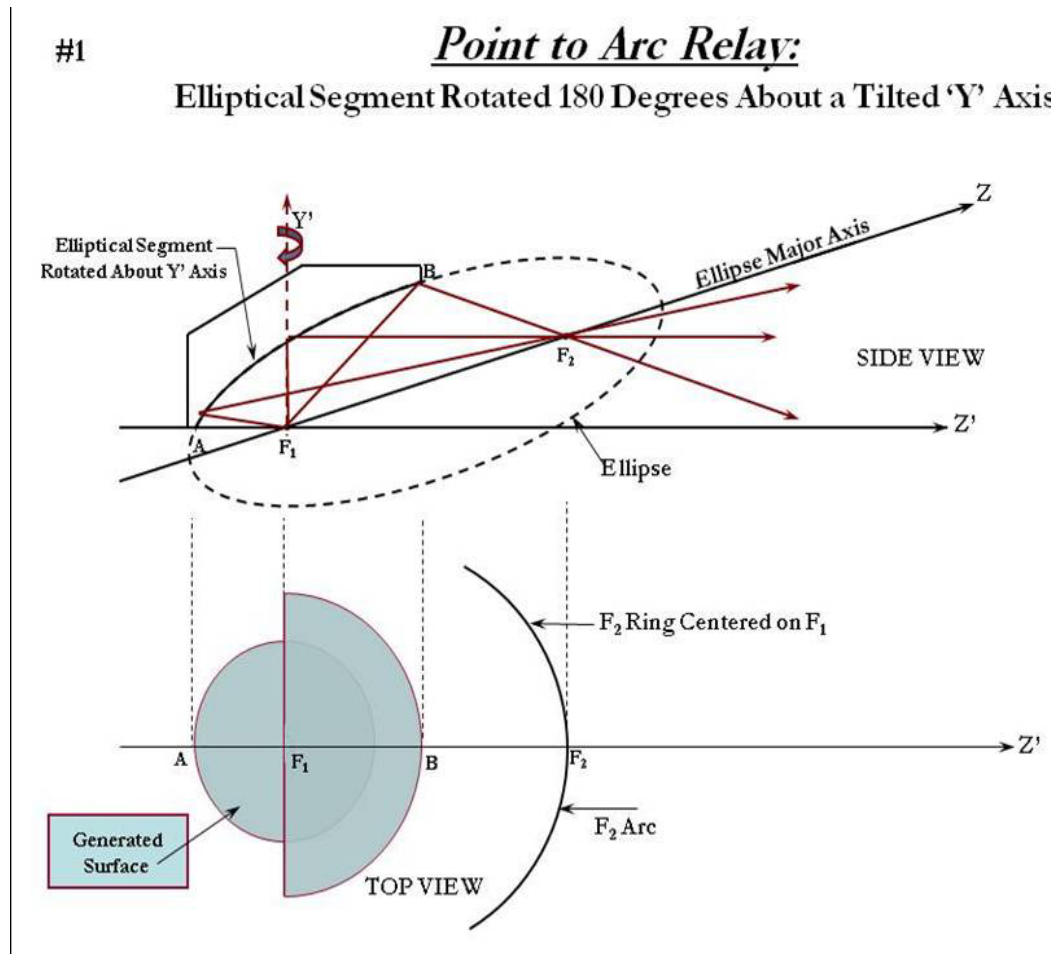

Figure 2a. The Point-to-Arc Generator is created by rotating a segment from a tilted ellipse about an axis that passes through the left side foci. Points $A, B, F_{1}$ and $F_{2}$ are dropped down from the side view to the top view for clarity.

Note that the arc image does not form a complete circle and is limited to 180 degrees.

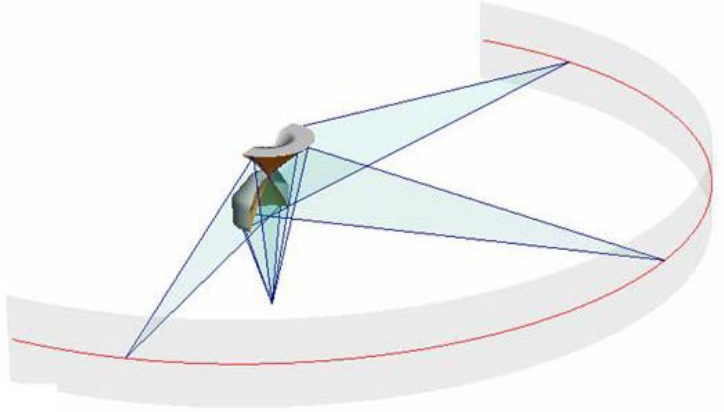

Figure 2b. Point-2-Arc Generator perspective view shows how the solid model is pinched to a point where the rotation axis intersects the elliptical segment. 


\subsection{Point-to-Ring Generator}

The Point-to-Ring Generator is another interesting transformer from an elliptical segment. This time however, the axis of rotation does not pass through the optical segment. The rotation axis is essentially in contact with the elliptical profile and passes through one of the foci. Because it does not intersect the optical surface, it can be swept through a full $2 \pi$ radians to form a solid circle, or ring of light. Figure $3 a$ and $3 b$ show the side and top view of this geometry. A point source at the stationary focal point $\mathrm{F}_{1}$, images on to a ring of light centered on the point source. Note that the NA of the source is symmetric while the NA of the image is non zero only in the side view plane (YZ).

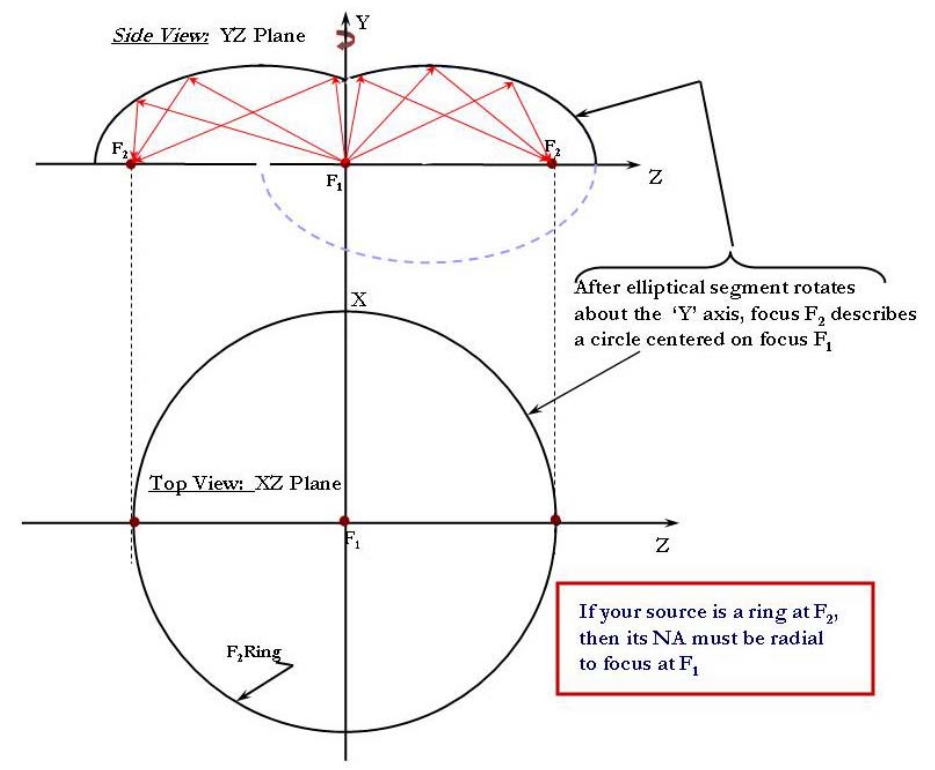

Figure 3a. Side view and top view of the Point-to-Ring Generator.
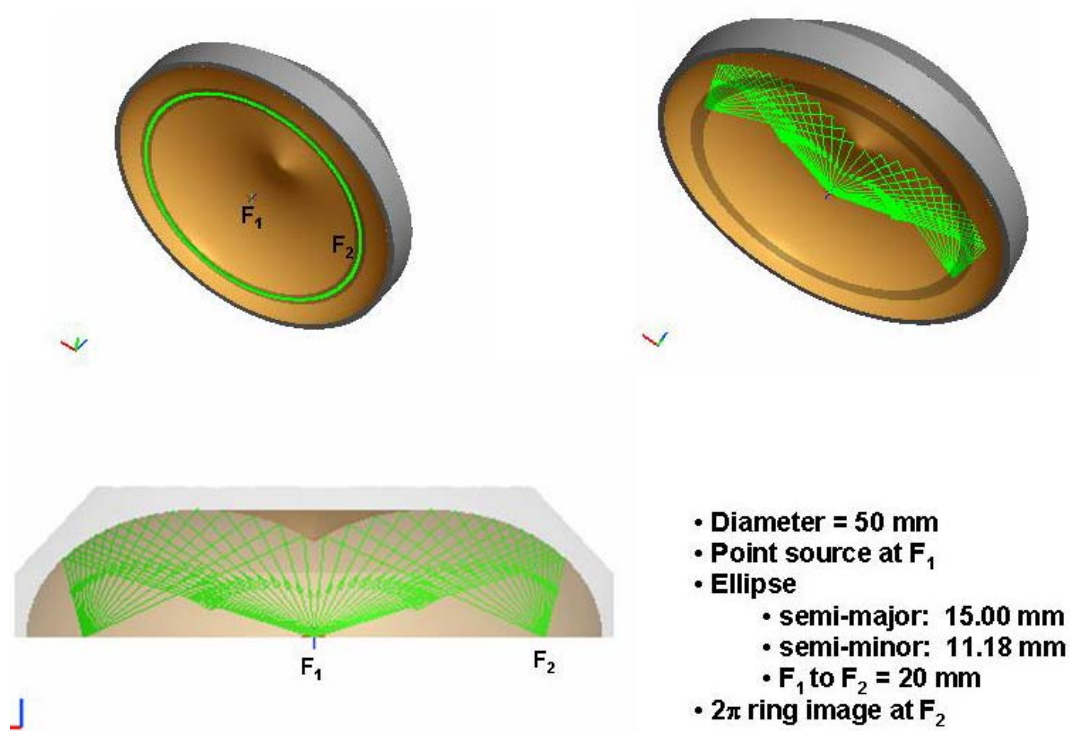

Figures 3b. Solid model $\left(\right.$ FRED $\left.^{1}\right)$ showing a radial fan of rays from $F_{1}$, imaged at $F_{2}$. 

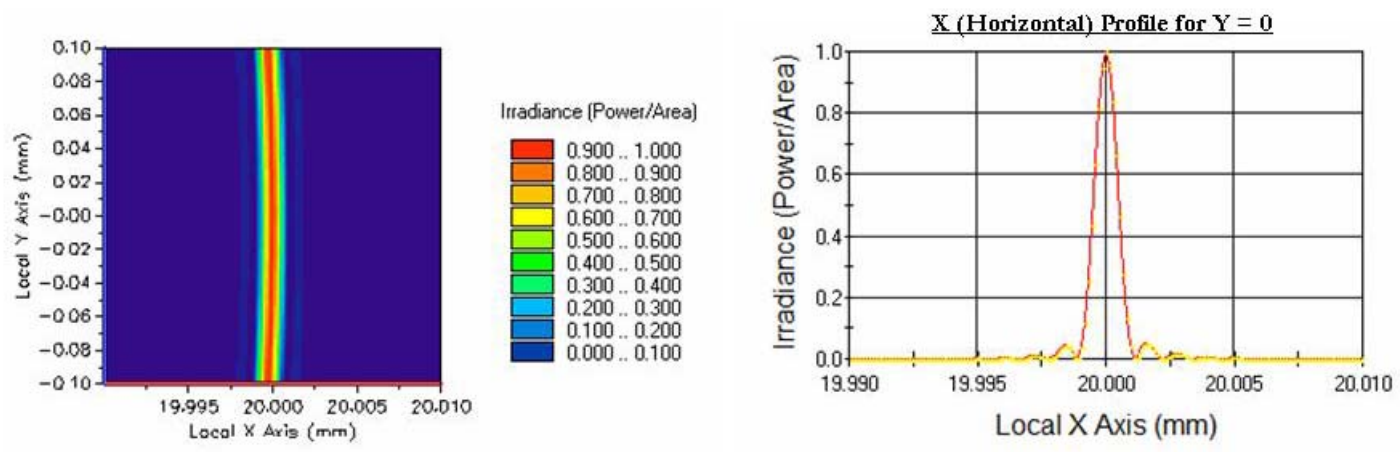

- Coherent point source at $F_{1}$

- Portion of diffraction PSF at $F_{2}(x=20 \mathrm{~mm})$

- normalized to unity

- note anamorphic scale in $\mathrm{X}$ and $\mathrm{Y}$

Figure 3c. PSF across the width of the ring image for the Point-2-Ring Generator.
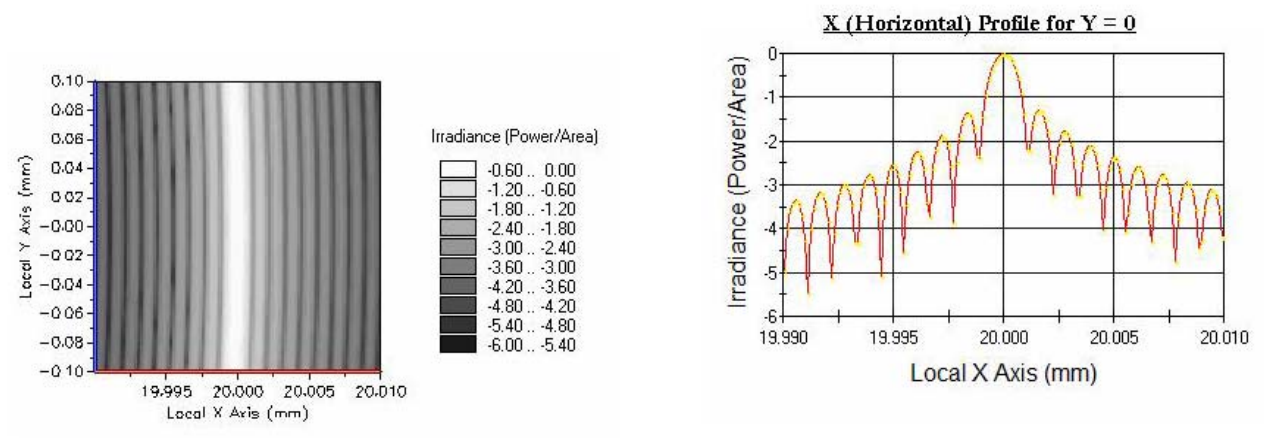

- Coherent point source at $F_{1}$

- Portion of diffraction PSF at $F_{2}(x=20 \mathrm{~mm})$

- Log-normalized

- note slight asymmetry left to right

- note anamorphic scale in $\mathrm{X}$ and $\mathrm{Y}$

Figure 3d. Log plot of PSF across width of ring image.

\subsection{Other Transformers}

Other transformers may be obtained by using a hyperbola as opposed to a parabola or ellipse. With a hyperbola, we get the opportunity to create virtual arc images. Figures $4 \mathrm{a}$ and $4 \mathrm{~b}$ show two variations of a theme using hyperbolas. 


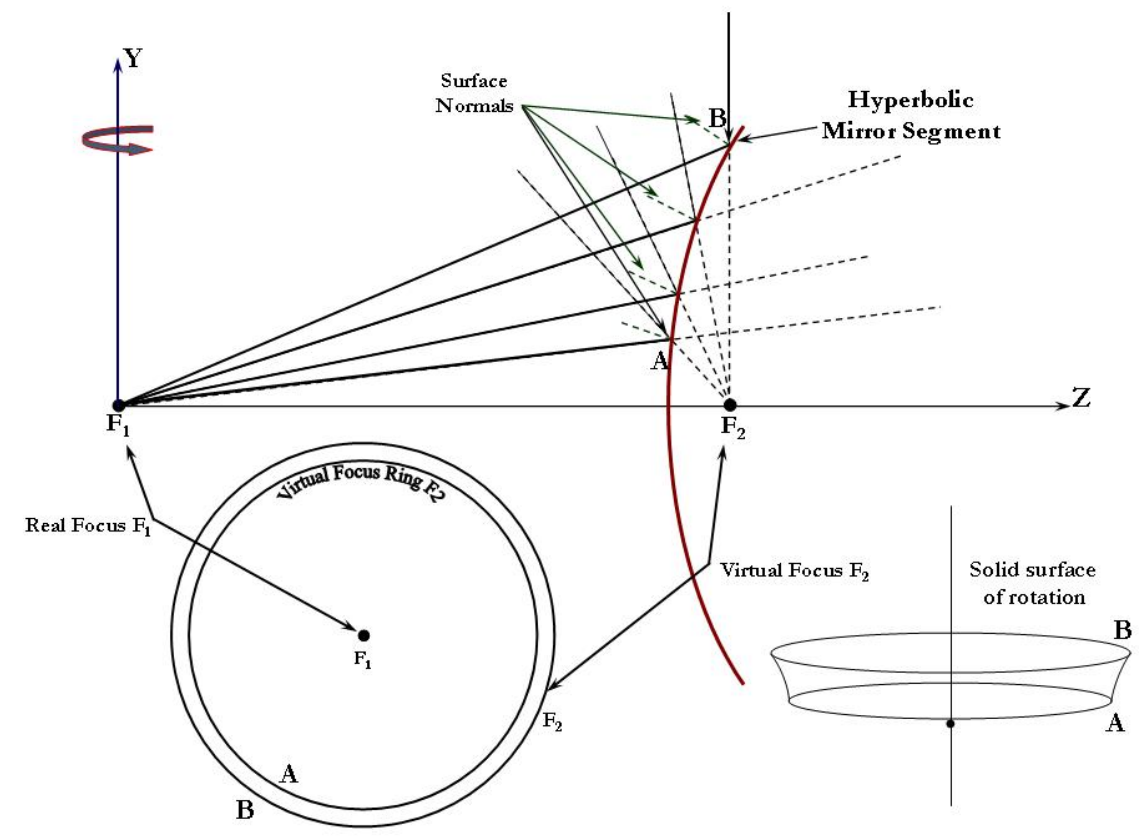

Figure 4a. Point-to-Virtual Ring Generator. A hyperbolic segment rotated about one of its focal points $\mathrm{F}_{1}$, will create a toroidal reflective ring and transform a point source $\left(a t F_{1}\right)$ to a virtual ring of light coming from $F_{2}$.

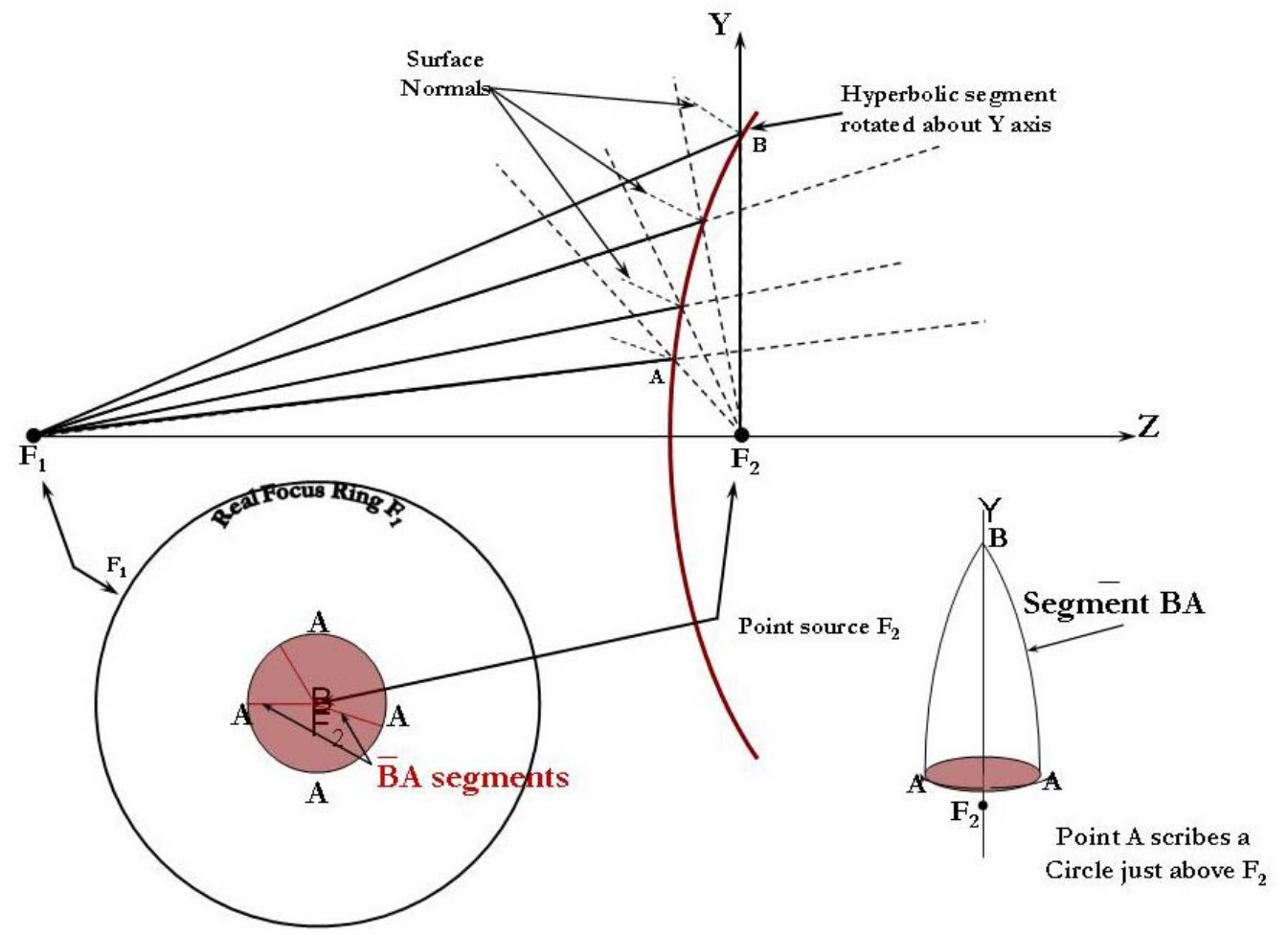

Figure 4b. A Virtual Point-to-Ring Generator will intercept converging light on to $F_{2}$ and form a ring image at $F_{1}$. 


\subsection{Examples in the real world}

The idea for these transformers came about during a task to focus LASER light on to the Grating Light Valve ${ }^{2}$ (GLV), to be used as a digital projector. The GLV is a programmable MEMS diffraction grating that is 25mm long and about $0.010 \mathrm{~mm}$ wide. LASER illumination was required to put enough light through such a narrow aperture. Hence, the Point-to-Line Generator was designed as an efficient and cost effective means to accomplish this task. Not only did this device provide a single surface solution to the problem, but the NA at the line image was in the correct plane to work with the diffraction of the device. The GLV diffraction angle is in the orthogonal plane relative to the incoming NA of the illuminating light and hence, the diffracted light can be blocked to prevent it from exiting the system to turn pixels on and off.

An interesting, non optical application of the Point-to-Arc Generator can be found as an acoustic lens in the Beo5 $5^{3}$ speaker system by Bang \& Olfusen. Figures $5 a$ and $5 b$ show how B\&O used a variation of the designs presented here to spread out the acoustic wavefront from a pair of speakers. A driver (tweeter or mid range) is located at the stationary focal point $F_{1}$. The acoustic lens forms an image of the driver at $F_{2}$, which is spread through a 180 degree arc, parallel to the floor and with little divergence in the up and down direction.

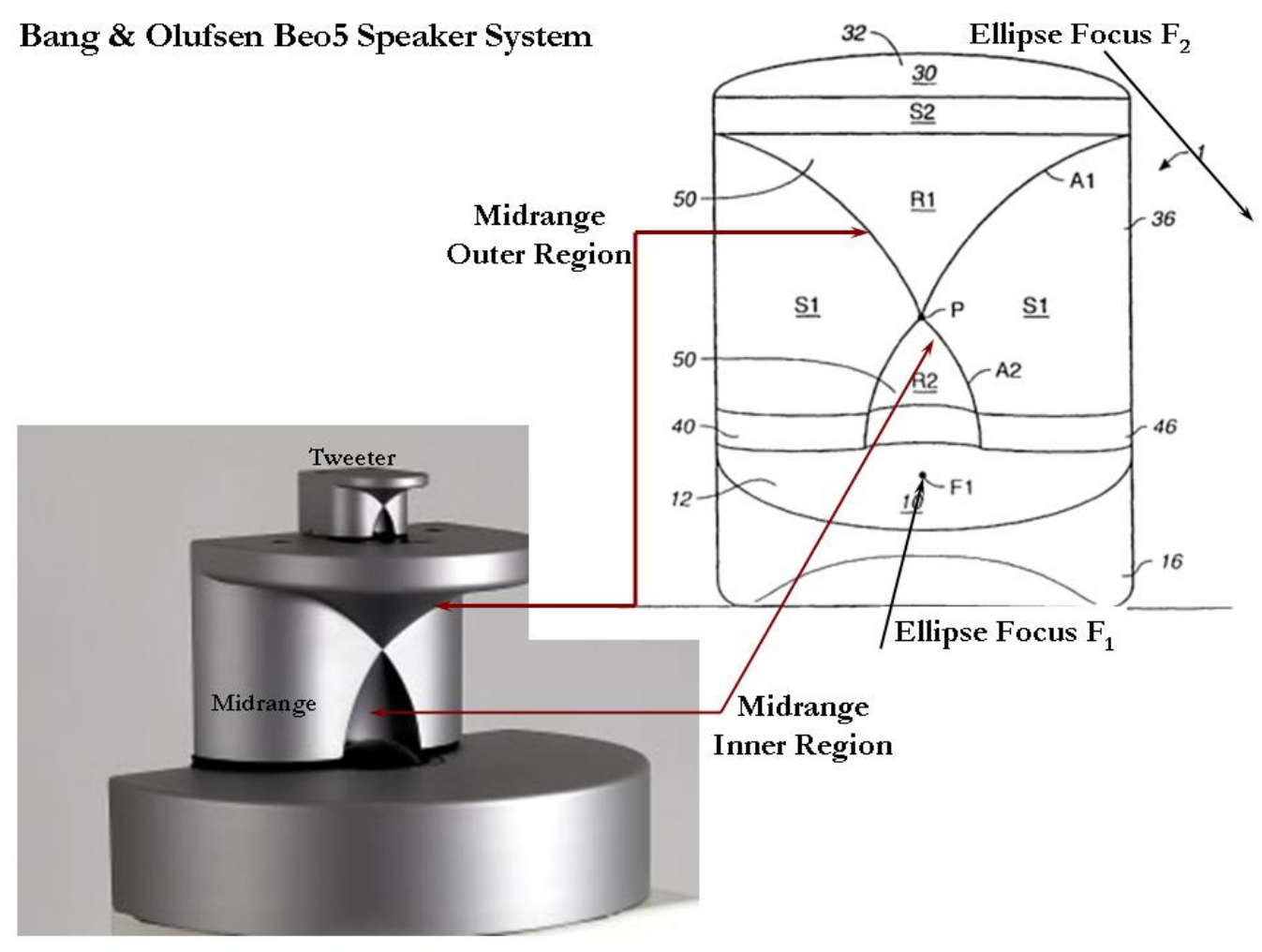

Figure 5a. Bang \& Olufsen Beo5 Speaker design using a version of the Point-to-Arc Generator described in this paper. 

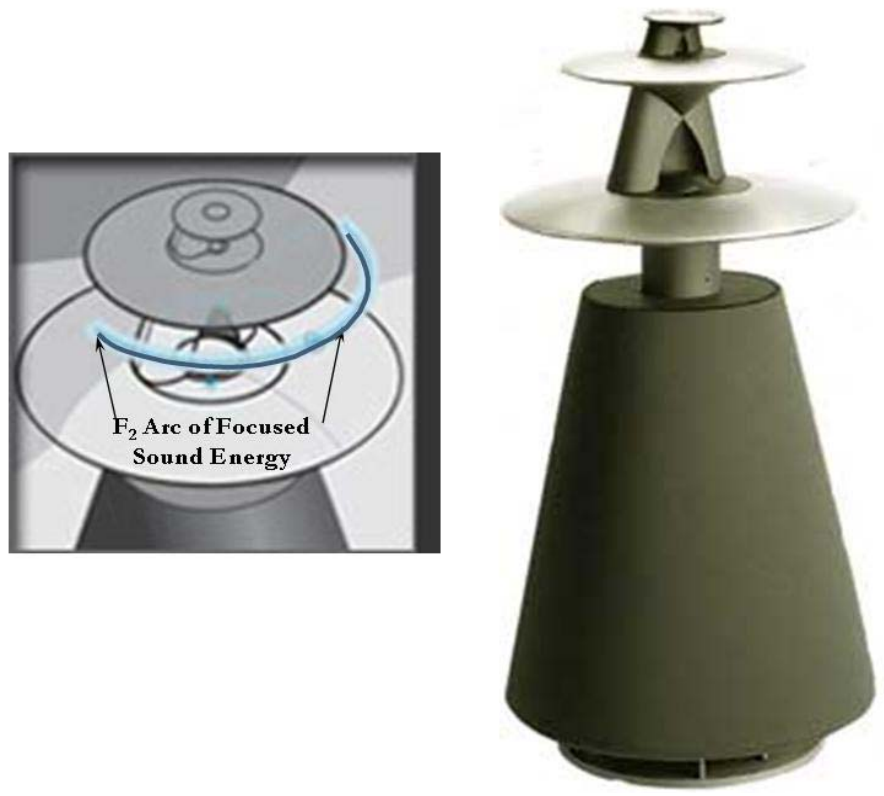

Figure 5b. B\&O acoustic lens showing the arc of acoustic energy at $\mathrm{F}_{2}$.

Another real world example of using Swept Conics can be found in the design of the Lightcraft ${ }^{4}$ device whose goal is to use LASER energy to launch small satellites in low earth orbit. Several photos of this small nosecone can be seen in Figures 6a through 6c. The collimated LASER energy from the ground hits the rotationally symmetric reflector and focuses that energy on to a ring around the perimeter. The back side of the craft is a large, highly polished parabolic mirror that is designed to capture the laser beam projected at it from the ground. The mirror focuses the beam, rapidly heating the air to 5 TIMES the temperature of the sun, creating a blast wave out the back that pushes the vehicle upward. As the beam is rapidly pulsed, the vehicle is continuously propelled forward, on its way to orbit.

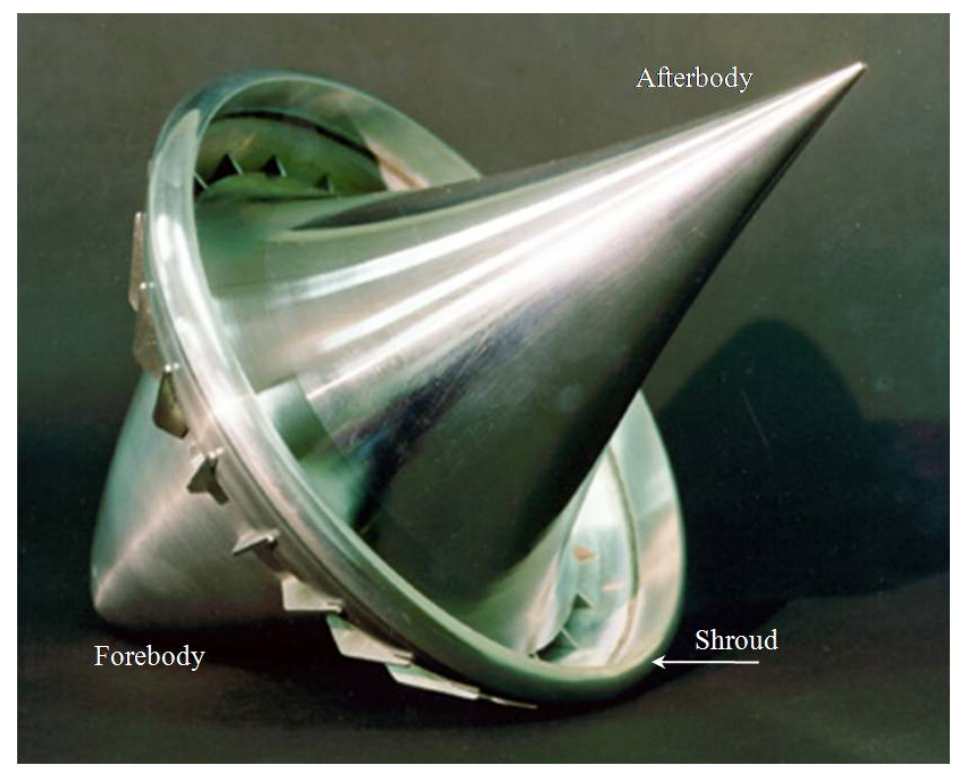

Figure 6a. Lightcraft nosecone showing the forebody for lift and compression, the shroud (cowl) around the perimeter where the LASER heating would take place, and the afterbody with mirrored surface for focusing LASER energy into the shroud. 


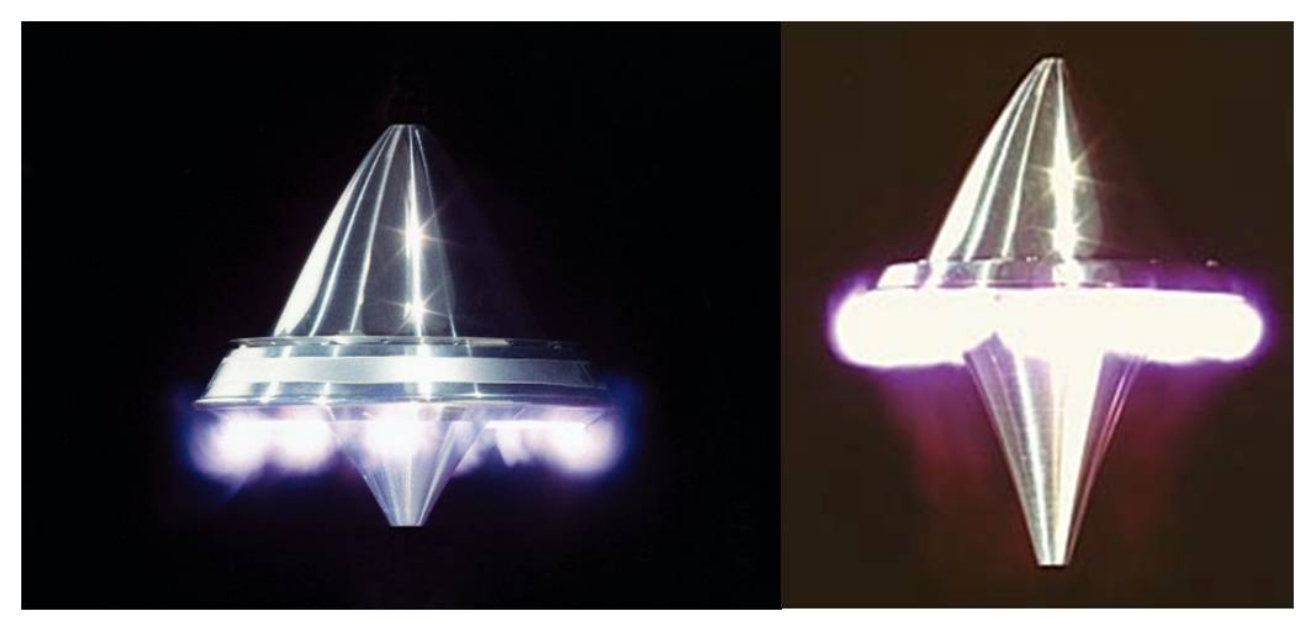

Figures 6b and 6c. A small LASER propelled nose cone receives collimated optical energy from a LASER below. The mirror focuses the beam, rapidly heating the air to 5 TIMES the temperature of the sun, creating a blast wave out the shroud that pushes the vehicle upward.

\subsection{Conclusion}

Several fascinating, single surface optical transformers have been demonstrated using symmetry and the optical properties of conical segments. By nailing down one of the foci of a conical segment and rotating said segment about an axis passing through the fixed foci, one can generate some useful and robust reflective optical surfaces that offer unique solutions to many optical and non-optical problems.

\section{References}

1. FRED is a proprietary product of Photon Engineering LLC, Tucson, AZ, www.photonengr.com

2. GLV is technology developed by Silicon Light Machines, Sunnyvale, CA, www.siliconlight.com

3. Beo5 is a product of Bang \& Olufsen, Norfolk, VA, www.bang-olufsen.com

4. Lightcraft is a developing technology of Lightcraft Technologies Incorporated, Bennington, VT, www.lightcrafttechnologies.com 